\title{
THE KINEMATICS OF THE LOCAL GROUP IN A COSMOLOGICAL CONTEXT
}

\author{
J. E. Forero-Romero ${ }^{1}$, Y. Hoffman ${ }^{2}$, S. Bustamante ${ }^{3}$, S. Gottlöber ${ }^{4}$, and G. YePes $^{5}$ \\ ${ }^{1}$ Departamento de Física, Universidad de los Andes, Cra. 1 No. 18A-10, Edificio Ip, Bogotá, Colombia; je.forero@uniandes.edu.co \\ ${ }^{2}$ Racah Institute of Physics, The Hebrew University of Jerusalem, 91904 Jerusalem, Israel \\ ${ }^{3}$ Instituto de Física-FCEN, Universidad de Antioquia, Calle 67 No. 53-108, Medellín, Colombia \\ ${ }^{4}$ Leibniz-Institut für Astrophysik, Potsdam, An der Sternwarte 16, D-14482 Potsdam, Germany \\ ${ }^{5}$ Grupo de Astrofísica, Departamento de Física Teórica, Universidad Autónoma de Madrid, Cantoblanco E-280049, Spain \\ Received 2013 February 15; accepted 2013 March 6; published 2013 March 21
}

\begin{abstract}
Recent observations constrained the tangential velocity of M31 with respect to the Milky Way to be $v_{\mathrm{M} 31, \tan }<$ $34.4 \mathrm{~km} \mathrm{~s}^{-1}$ and the radial velocity to be in the range $v_{\mathrm{M} 31 \text {, rad }}=-109 \pm 4.4 \mathrm{~km} \mathrm{~s}^{-1}$. In this study we use a large volume high-resolution $N$-body cosmological simulation (Bolshoi) together with three constrained simulations to statistically study this kinematics in the context of the $\Lambda$ cold dark matter ( $\Lambda \mathrm{CDM})$. The comparison of the ensembles of simulated pairs with the observed Local Group (LG) at the $1 \sigma$ level in the uncertainties has been done with respect to the radial and tangential velocities, the reduced orbital energy $\left(e_{\text {tot }}\right)$, angular momentum $\left(l_{\text {orb }}\right)$, and the dimensionless spin parameter, $\lambda$. Our main results are (1) the preferred radial and tangential velocities for pairs in $\Lambda \mathrm{CDM}$ are $v_{\mathrm{r}}=-80 \pm 20 \mathrm{~km} \mathrm{~s}^{-1}$ and $v_{\mathrm{t}}=50 \pm 10 \mathrm{~km} \mathrm{~s}^{-1}$, (2) pairs around that region are 3-13 times more common than pairs within the observational values, (3) 15\%-24\% of LG-like pairs in $\Lambda$ CDM have energy and angular momentum consistent with observations, while (4) $9 \%-13 \%$ of pairs in the same sample show similar values in the inferred dimensionless spin parameter. It follows that within current observational uncertainties the quasi-conserved quantities that characterize the orbit of the LG, i.e., $e_{\text {tot }}, l_{\text {orb }}$, and $\lambda$, do not challenge the standard $\Lambda \mathrm{CDM}$ model, but the model is in tension with regard to the actual values of the radial and tangential velocities. This might hint to a problem of the $\Lambda \mathrm{CDM}$ model to reproduce the observed LG.
\end{abstract}

Key words: galaxies: kinematics and dynamics - Local Group - methods: numerical

\section{INTRODUCTION}

The Milky Way (MW) and Andromeda galaxy (M31) are the dominant galaxies in the Local Group (LG). Astronomical observations of their mass distribution impose constraints on the standard cosmological model. The satellite overabundance problem (Klypin et al. 1999b; Moore et al. 1999), tidal disruption features (McConnachie et al. 2009), and the disk-dominated morphology (Kazantzidis et al. 2008) are examples of how LG studies are linked to the cosmological context. Detailed studies on the Magellanic Clouds dynamics and their possible link to M31 add to the interest of understanding the details of the LG kinematics and dynamics (Besla et al. 2007; Tollerud et al. 2011; Knebe et al. 2011; Fouquet et al. 2012; Teyssier et al. 2012). However, a general concern in the use of the LG as a tool for near-field cosmology (Freeman \& Bland-Hawthorn 2002; Peebles \& Nusser 2010) is how typical is the LG regarding the properties of interest (Busha et al. 2011; Liu et al. 2011; Forero-Romero et al. 2011; Purcell \& Zentner 2012).

A new valuable piece of information in this issue is the recent observational determination of the proper-motion measurements of M31, which until recently had been out of reach (van der Marel et al. 2012). The reported measurements set an upper bound for the tangential velocity of M31 with respect to the MW of $v_{\tan , \mathrm{M} 31} \leqslant 34.4 \mathrm{~km} \mathrm{~s}^{-1}$. Together with the values of the relative radial velocity of $v_{\text {rad,M31 }}=-109 \pm 4.4 \mathrm{~km} \mathrm{~s}^{-1}$, observations show that the relative motion of the MW and Andromeda is consistent with a head-on collision. With this information, it is possible to quantify how common such a kinematic configuration is in a $\Lambda$ cold dark matter $(\Lambda \mathrm{CDM})$ universe.

This Letter presents such a study. We use a large volume, high-resolution-dark-matter-(DM)-only $N$-body simulation in the concordance $\Lambda \mathrm{CDM}$ cosmology to find a set of halo pairs with similar characteristics as inferred in the LG. We quantify these results in terms of the number of pairs with given radial and tangential velocities in the galactocentric rest frame. We also find the pairs that are consistent with a headon collision in terms of the ratio of the radial to tangential velocity $f_{\mathrm{t}} \equiv v_{\mathrm{tan}} / v_{\mathrm{rad}}<0.32$ and present these results in terms of the reduced angular momentum, mechanical energy, and dimensionless spin parameter.

In addition, we make use of three constrained $N$-body simulations which are constructed to reproduce the observed large-scale structure of the Local Universe on scales of a few tens of Mpc. The special feature of these simulations is that each volume features a pair of halos with the right characteristics to be considered LG-like objects.

This Letter is structured as follows. In the next section we present the $N$-body simulations and the criteria we use to select LG-like halo pairs. In Section 3 we present the results for the dynamics in these pairs in terms of the tangential/ radial velocities and the orbital angular momentum/mechanical energy. In the same section we summarize these dynamical results in terms of the dimensionless spin parameter of the pairs. Finally, in the last section we comment and conclude about the implications of these results in the context of the $\Lambda \mathrm{CDM}$ model.

\section{SIMULATION AND PAIR SAMPLES}

\subsection{The Bolshoi and Constrained Simulations}

The Bolshoi simulation follows the non-linear evolution of the DM density field using $N$-body techniques. The simulation has a cubic comoving volume of $\left(250 h^{-1} \mathrm{Mpc}\right)^{3}$, sampled with $2048^{3}$ particles. The cosmological parameters used in the simulation are $\Omega_{m}=0.27, \Omega_{\Lambda}=0.73, \sigma_{8}=0.82, h=0.70$, and $n=0.95$, corresponding to the matter density, vacuum 
energy density, the normalization of the power spectrum, the dimensionless Hubble constant, and the index of the slope in the initial power spectrum. This set of parameters is compatible with the analysis of the seventh year of data from the Wilkinson Microwave Anisotropy Probe (WMAP; Jarosik et al. 2011). A detailed description of this simulation can be found in Klypin et al. (2011).

With these parameters the mass per particle is $m_{p}=1.4 \times$ $10^{8} h^{-1} M_{\odot}$. In this Letter we use halos obtained through the Bound Density Maxima (BDM) algorithm (Klypin et al. 1999a). The halos are selected to have an overdensity of 200 times the critical density. Furthermore, we only include in the analysis halos whose center is located outside the virial radius of any other halo. We have obtained the data through the publicly available Multidark database ${ }^{6}$ (Riebe et al. 2011). The database allows us to obtain the comoving positions, peculiar velocities, and masses for all the halos in the simulation volume at $z=0$. The positions and velocities of these haloes correspond to the average values of the 250 most bound particles. The Hubble flow is taken into account to convert the peculiar velocities into physical velocities and allow for a comparison with observations. We have verified that the main conclusions of this Letter hold in the case of halos defined by a Friendsof-Friends (FoF) algorithm with a linking length 0.17 times the mean interparticle distance.

The constrained simulations we use in this Letter are part of the Constrained Local UniversE Simulations (CLUES) ${ }^{7}$ project whose main objective is to reproduce the large-scale structure in the Local Universe as accurately as possible. The algorithm and observational constraints to construct the initial conditions are described in Gottlöber et al. (2010). We use three DMonly simulations, each has a cubic volume of $64 h^{-1} \mathrm{Mpc}$ on a side, with the density field sampled with $1024^{3}$ particles. The cosmological density parameter is $\Omega_{m}=0.28$, the cosmological constant $\Omega_{\Lambda}=0.72$, the dimensionless Hubble parameter $h=$ 0.73 , the spectral index of the primordial density perturbations $n=0.96$, and the power spectrum normalization $\sigma_{8}=0.817$, also consistent with WMAP 7th year data.

\subsection{Two Samples of LG-like Pairs}

Based on the BDM catalogs in the Bolshoi simulation, we construct a halo pair sample with the dynamical properties consistent with those of the MW and M31. The criteria we impose to define a LG-like halo pair are the following.

1. Each halo has a mass in the range $7 \times 10^{11} M_{\odot}<M_{h}<$ $7 \times 10^{12} M_{\odot}$.

2. With respect to each halo, there cannot be any other halo within the mass range $7 \times 10^{11} M_{\odot}<M_{h}<7 \times 10^{12} M_{\odot}$ closer than its partner. It means that there cannot be ambiguity on the identity of the pair members.

3. The relative radial velocity between the two halos is negative (van der Marel et al. 2012).

4. The distance between the center of mass of the halos must be less than 1.0 Mpc (Ribas et al. 2005; van der Marel \& Guhathakurta 2008).

5. There cannot be halos more massive than $7 \times 10^{12} M_{\odot}$ within a radius of $3 \mathrm{Mpc}$ with respect to every object center (Karachentsev et al. 2004; Tikhonov \& Klypin 2009).

\footnotetext{
6 http://www.multidark.org/MultiDark/

7 http://www.clues-project.org
}

Table 1

Summary of Kinematic Observational Constraints

\begin{tabular}{lcc}
\hline \hline$v_{\mathrm{M} 31, \mathrm{rad}}$ & $\left(\mathrm{km} \mathrm{s}^{-1}\right)$ & $-109.3 \pm 4.4$ \\
\hline$v_{\mathrm{M} 31, \tan }$ & $\left(\mathrm{km} \mathrm{s}^{-1}\right)$ & $<34.4$ \\
$r_{\mathrm{M} 31}$ & $(\mathrm{kpc})$ & $770 \pm 40$ \\
$\mathbf{r}_{\mathrm{M} 31}$ & $(\mathrm{kpc})$ & $(-378.9,612.7,-283.1)$ \\
$\sigma_{\mathbf{r}, \mathrm{M} 31}$ & $(\mathrm{kpc})$ & $(-18.9,30.6,14.5)$ \\
$\mathbf{v}_{\mathrm{M} 31}$ & $\left(\mathrm{~km} \mathrm{~s}^{-1}\right)$ & $(66.1,-76.3,45.1)$ \\
$\sigma_{\mathbf{v}, \mathrm{M} 31}$ & $\left(\mathrm{~km} \mathrm{~s}^{-1}\right)$ & $(26.7,19.0,26.5)$ \\
$M_{200, \mathrm{MW}}$ & $\left(10^{12} M_{\odot}\right)$ & $1.6 \pm 0.5$ \\
$M_{200, \mathrm{M} 31}$ & $\left(10^{12} M_{\odot}\right)$ & $1.6 \pm 0.5$ \\
$M_{200, \mathrm{MW}}+M_{200, \mathrm{M} 31}$ & $\left(10^{12} M_{\odot}\right)$ & $3.14 \pm 0.58$ \\
$\log _{10} \lambda$ & & $-1.72 \pm 0.07$ \\
\hline
\end{tabular}

Notes.

1. The kinematic properties for M31 are reported in the galactocentric rest frame (van der Marel et al. 2012).

2. Values in parenthesis correspond to vector components. $\sigma_{\mathbf{x}}$ represents the uncertainty on the components of vector $\mathbf{x}$. The uncertainties correspond to $1 \sigma$ values.

3. The values for the individual halo masses are consistent with the priors used by van der Marel et al. (2012).

4. The observational uncertainties in the position vector correspond to a $5 \%$ in each component consistent with the $1 \sigma$ uncertainties in the distance (see references in van der Marel \& Guhathakurta 2008).

5. The value for $\log _{10} \lambda$ is obtained in this Letter from a Monte Carlo simulation as described in Section 3.

6. There cannot be halos more massive than $7 \times 10^{13} M_{\odot}$ within a radius of $4 \mathrm{Mpc}$ with respect to every object center (Karachentsev et al. 2004).

Throughout this Letter we refer to this sample as the full sample. This sample in the Bolshoi simulation has 1923 pairs. Additionally, there is a sample of three pairs constructed from the three constrained realizations. These pairs fulfill all the above-mentioned conditions and additionally are located in a place with the right distances with respect to the Virgo cluster in the simulation.

The full observational characteristics that we take in this Letter for the MW-M31 pair are listed in Table 1. A more reduced sample from the full sample has been constructed so as to obey the observational bounds on the masses and separation of the two main halos. These amount to the following.

1. The separation between the center of mass of the halos is in the range 700-800 kpc (Ribas et al. 2005; van der Marel \& Guhathakurta 2008).

2. The total mass of the two halos is in the range $1-4 \times 10^{12} M_{\odot}$ (van der Marel et al. 2012).

Including these conditions the full sample is reduced from 1923 to 158 pairs. We refer to this sample as the reduced sample. Note that only one constrained LG-like object is included in this subset.

\section{RESULTS}

\subsection{Radial and Tangential Velocities}

Figure 1 summarizes the central finding of this Letter. Most of the pairs in the two samples constructed from the Bolshoi Simulation have radial and tangential velocities notably different from the observational constraints at the $1 \sigma$ level.

The most probable radial and tangential velocities in $\Lambda \mathrm{CDM}$ are summarized in Table 2. For the full sample, we have $v_{\text {rad, } \Lambda \mathrm{CDM}}=-70 \pm 10 \mathrm{~km} \mathrm{~s}^{-1}$ and $v_{\mathrm{tan}, \Lambda \mathrm{CDM}}=50 \pm 20 \mathrm{~km} \mathrm{~s}^{-1}$. 

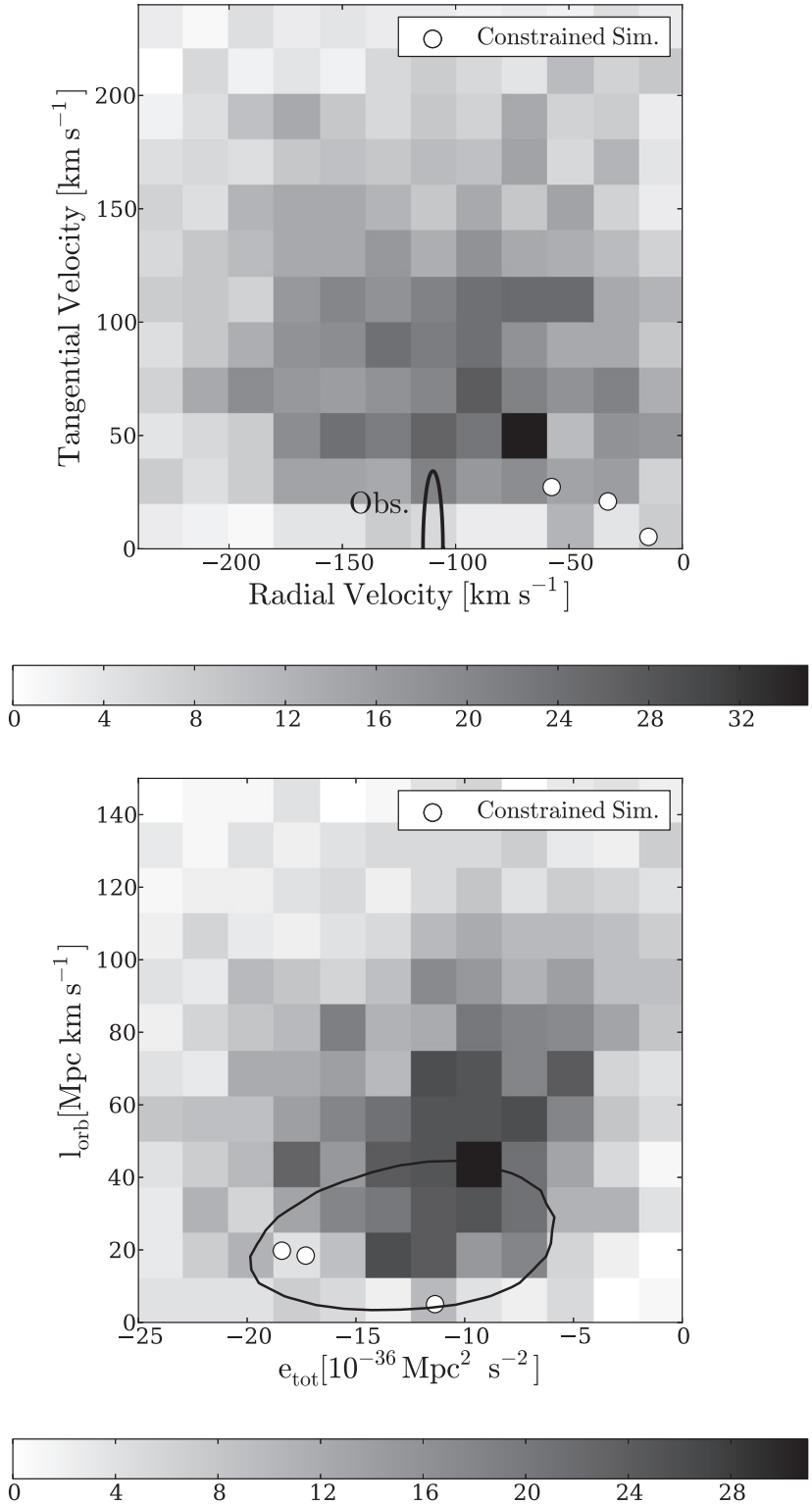
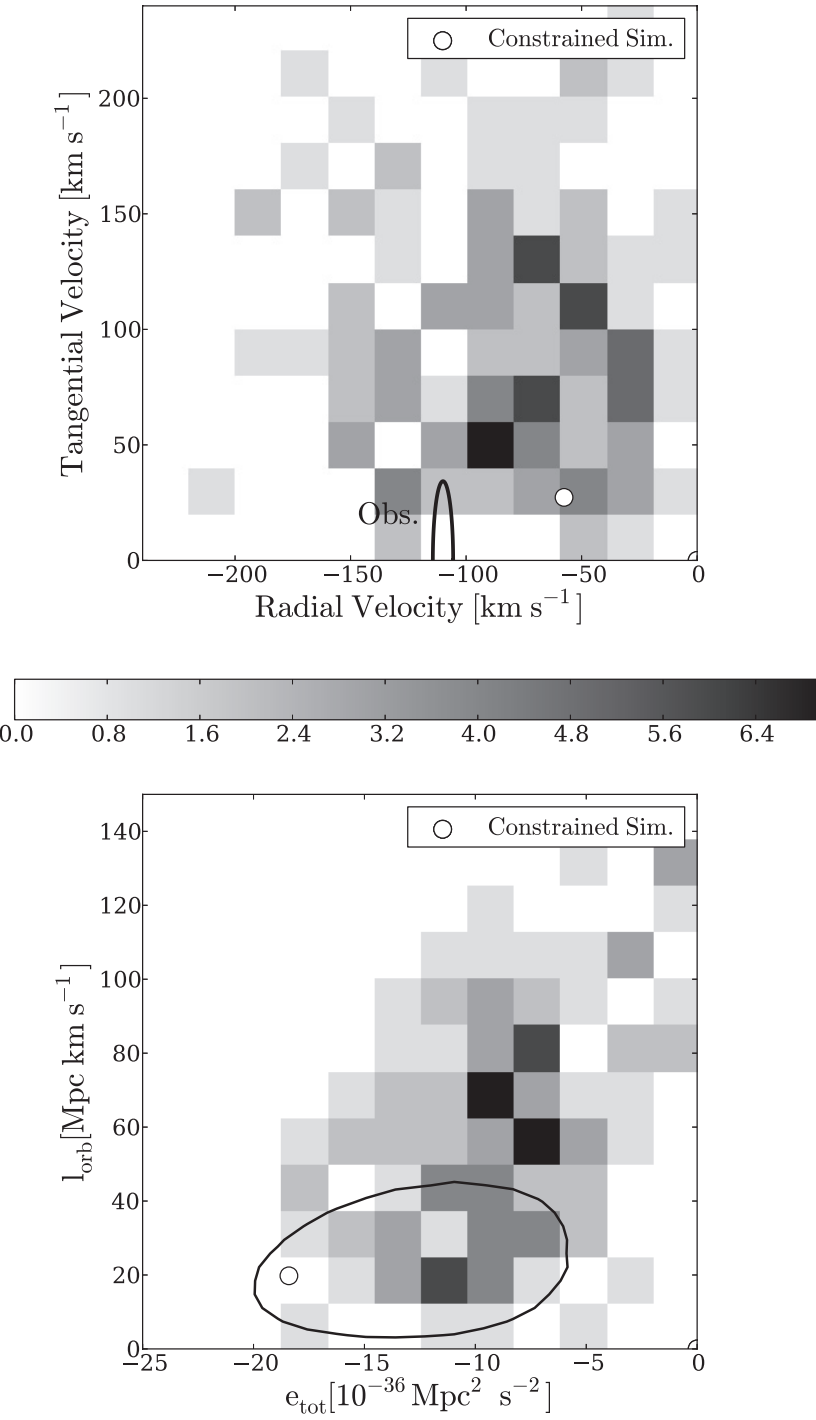

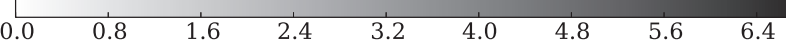

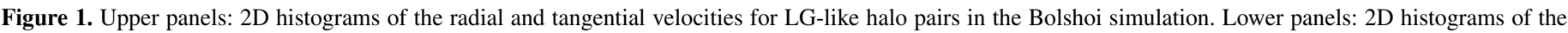

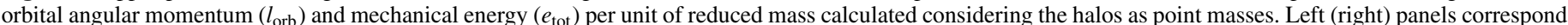

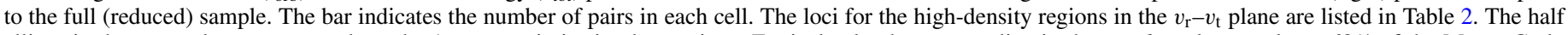

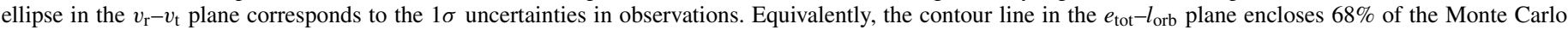
generated points from the observational values summarized in Table 1 . The circles represent the positions of the pairs from the constrained simulations.

Table 2

Summary of Results from the $\Lambda$ CDM Bolshoi Simulation

\begin{tabular}{lcc}
\hline \hline & Full Sample & Reduced Sample \\
\hline$v_{\mathrm{M} 31, \operatorname{rad}}\left(\mathrm{km} \mathrm{s}^{-1}\right)$ & $-70 \pm 10$ & $-90 \pm 10$ \\
$v_{\mathrm{M} 31, \tan }\left(\mathrm{km} \mathrm{s}^{-1}\right)$ & $50 \pm 10$ & $50 \pm 10$ \\
$\log _{10} \lambda$ & $-1.47 \pm 0.13$ & $-1.34 \pm 0.12$ \\
\hline
\end{tabular}

Note. The velocity uncertainties correspond to the minimum bin size required to obtain robust statistics in Figure 1.

For the reduced sample, $v_{\text {rad, }} \Lambda \mathrm{CDM}=-90 \pm 10 \mathrm{~km} \mathrm{~s}^{-1}$ and $v_{\text {tan, }, \Lambda \mathrm{CDM}}=50 \pm 20 \mathrm{~km} \mathrm{~s}^{-1}$, where the uncertainties in these values reflect the minimum grid size needed to obtain robust statistics for the two-dimensional (2D) histogram.

The number of pairs compatible with LG observations at the $1 \sigma$ level is listed in Table 3 (Columns 2 and 3). In the same table (Columns 4 and 5) we summarize the number of pairs around the
$\Lambda \mathrm{CDM}$ values within the same range of absolute observational uncertainty (i.e., $\sigma_{\mathrm{tan}}=17 \mathrm{~km} \mathrm{~s}^{-1}$ and $\sigma_{\mathrm{rad}}=4 \mathrm{~km} \mathrm{~s}^{-1}$ ).

From these results we infer that the pairs around the preferred phase space for $\Lambda \mathrm{CDM}$ are at least 13 times more common than pairs with the observed velocities for the LG. We highlight that this is a lower bound given that in the reduced sample none of the pairs are found in the interval allowed by observations. The high eccentric orbit of the observed LG constitutes an unlikely configuration for the $\Lambda \mathrm{CDM}$ LG-like objects. This holds for the full and the reduced sample of objects. These conclusions are valid at $1 \sigma$ level in the observational uncertainties. An equivalence in the abundance between the pairs around the observational values and those around the preferred $\Lambda \mathrm{CDM}$ velocities is reached only at the $4 \sigma$ level on the observational uncertainties.

From Figure 1 it is also clear that there is a significant number of pairs with a high tangential-to-radial velocity ratio. The 

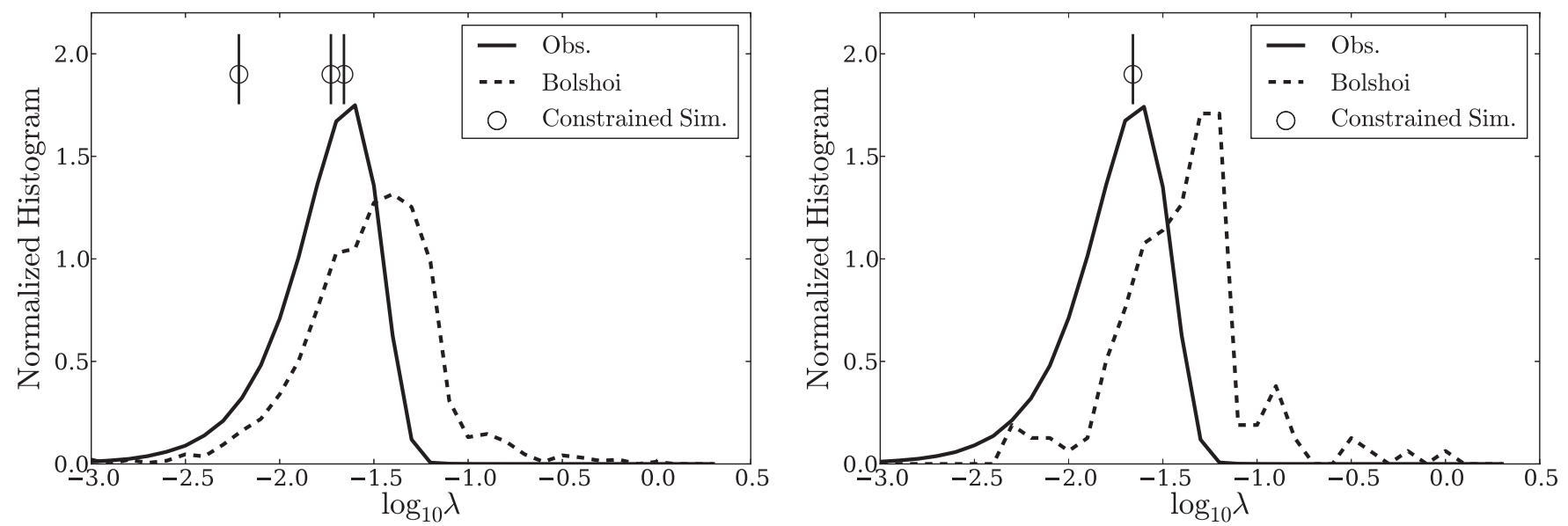

Figure 2. Normalized histograms of Peebles' spin parameter $\lambda$ for the pairs in the Bolshoi simulation and its inferred values for the LG from the observational constraints from a Monte Carlo simulation. The left (right) panel corresponds to the full (reduced) sample. The vertical lines with the white dots represent the values inferred for pairs in the constrained simulations.

Table 3

Summary of the Comparison of the Observational Results Against $\Lambda \mathrm{CDM}$

\begin{tabular}{lcccc}
\hline \hline $\begin{array}{l}\text { Physical } \\
\text { Property }\end{array}$ & $\begin{array}{c}\text { (\%) Pairs Consistent } \\
\text { with Observations }(1 \sigma) \\
\text { (Full Sample) }\end{array}$ & $\begin{array}{c}\text { (\%) Pairs Consistent } \\
\text { with Observations }(1 \sigma) \\
\text { (Reduced Sample) }\end{array}$ & $\begin{array}{c}\text { \%) Pairs with Highest } \\
\text { Likelihood in } \Lambda \text { CDM } \\
\text { (Full Sample) }\end{array}$ & $\begin{array}{c}(\%) \text { Pairs with Highest } \\
\text { Likelihood in } \Lambda \text { CDM } \\
(\text { Reduced Sample) }\end{array}$ \\
\hline$v_{\mathrm{r}}-v_{\mathrm{t}}$ & $(0.4 \%) 8 / 1923$ & $(<0.6 \%) 0 / 158$ & $(1 \%) 23 / 1923$ & $\ldots$ \\
$e_{\mathrm{tot}}-l_{\mathrm{orb}}$ & $(15 \%) 298 / 1923$ & $(24 \%) 38 / 158$ & $\ldots$ & $\ldots$ \\
$\log _{10} \lambda$ & $(13 \%) 257 / 1923$ & $(9 \%) 15 / 158$ & $\ldots$ & $\ldots$ \\
$r_{\mathrm{t}}=v_{\mathrm{t}} / v_{\mathrm{r}}$ & $(12 \%) 242 / 1923$ & $(8 \%) 13 / 158$ & $\ldots$ & $\ldots$ \\
\hline
\end{tabular}

Notes.

1. In Columns 2 and 3, consistency is defined from $1 \sigma$ uncertainties for $v_{\mathrm{r}}-v_{\mathrm{t}}, e_{\mathrm{tot}}-l_{\mathrm{orb}}$, and $\log _{10} \lambda$. In the case of $r_{\mathrm{t}}$, consistency is defined from the constraint $r_{\mathrm{t}}<0.32$ derived from the $1 \sigma$ observational uncertainties in the radial and tangential velocities.

2. In Columns 4 and 5, the number of pairs around the preferred region in $v_{\mathrm{r}}-v_{\mathrm{t}}$ in $\Lambda \mathrm{CDM}$ (as shown in Figure 1 and summarized in Table 2) is calculated using the same observational $1 \sigma$ absolute uncertainty on $v_{r}$ and $v_{t}$.

peak in the pair number density is located around a region of $f_{\mathrm{t}} \equiv v_{\tan \Lambda \mathrm{CDM}} / v_{\operatorname{rad} \Lambda \mathrm{CDM}} \sim 0.7$, while the observations suggest $f_{\mathrm{t}}<0.32$. As summarized in Table 3 , we find that only between $8 \%$ and $12 \%$ of the pairs are consistent with the observational constraint. The three pairs from the constrained realizations are also show higher $f_{\mathrm{t}}$ ratios than observations: $f_{\mathrm{t}}=0.35,0.45,0.73$.

\subsection{Reduced Angular Momentum and Energy}

The two-body problem of point-like masses can serve as a proxy for the dynamics of the LG and can be used as a tool for studying the LG within the framework of the standard cosmological model. Within the model the dynamics of the LG is governed by the (center of mass) reduced angular momentum $l_{\text {orb }}=\left|\mathbf{r}_{\mathrm{M} 31} \times \mathbf{v}_{\mathrm{M} 31}\right|$ and reduced energy $e_{\mathrm{tot}}=\left.(1 / 2) \mathbf{v}_{\mathrm{M} 31}\right|^{2}-$ $G M /\left|\mathbf{r}_{\mathrm{M} 31}\right|$, where the total mass is $M=m_{\mathrm{M} 31}+m_{\mathrm{MW}}$, the reduced mass is $\mu=m_{\mathrm{M} 31} m_{\mathrm{MW}} / M$, and $G$ is the gravitational constant.

This formulation has a clear theoretical advantage if one considers the angular momentum and the mechanical energy as quasi-conserved dynamical quantities. This means that, after some formation time, these quantities do not significantly evolve.

However, this formulation has an observational disadvantage. The reduced energy and angular momentum are derived from very different kinds of observations, increasing the uncertainties in their final determination.

We use Monte Carlo sampling to estimate the reduced energy and angular momentum from the observed properties in the LG listed in Table 1. The lower panels in Figure 1 also present the results in the plane $e_{\mathrm{tot}}-l_{\mathrm{orb}} \cdot{ }^{8}$ As expected, the $e_{\mathrm{tot}}$ and $l_{\mathrm{orb}}$ constraints are less restrictive compared with the radial and tangential velocity constraints; $15 \%(24 \%)$ of pairs in the full (reduced) sample obey the $1 \sigma$ observational constraints. We note that for the reduced sample the preferred region is outside the $1 \sigma$ observational contours.

All the constrained LGs of the full and reduced sample are consistent with the $1 \sigma$ observational uncertainty. However, a hypothetical increase by a factor of two in accuracy of the tangential velocity, showing that it is below $17 \mathrm{~km} \mathrm{~s}^{-1}$, would bring these pairs and the $\Lambda$ CDM expectation outside this uncertainty region.

\subsection{Dimensionless Spin Parameter}

The dimensionless spin parameter $\lambda$ (Peebles 1971) is used here to characterize the dynamical state of the observed and simulated LGs. The parameter measures the dynamical role of the angular momentum in terms of the gravitational attraction and is defined by

$$
\lambda=\frac{\mu^{3 / 2} l_{\mathrm{orb}} \sqrt{e_{\mathrm{tot}}}}{G M^{5 / 2}} .
$$

We compare the distribution for $\lambda$ obtained from the pair population in the Bolshoi simulation and the distribution from the Monte Carlo simulation used to estimate the uncertainties

\footnotetext{
8 The $1 \sigma$ contour in the lower panels of Figure 1 does not cross the zero level, as can be naively expected from Figure 1 . This happens for the $\approx 80 \%$ contour level.
} 
on $e_{\text {tot }}$ and $l_{\text {orb }}$. Figure 2 shows the likelihood distribution of the observational errors and the spin parameter of the LG-like pairs of the full (left panel) and the reduced (right panel) samples. The value of $\lambda$ of the constrained LGs is shown as well.

The expected observational value for $\lambda$ is slightly inconsistent with the statistics derived from halo pairs in $\Lambda$ CDM. The spin parameter of the simulated LG-like objects is skewed toward higher values compared with the observational estimation. Inspection of Figure 2 also shows that the $\lambda$ distribution is close to a lognormal one and thus resembles the distribution of the spin parameter of DM halos in simulation (Bett et al. 2007).

The estimated value of $\log _{10} \lambda$ of the observed LG is $-1.72 \pm$ 0.07 , lower than the mean value of $\log _{10} \lambda$ of $-1.47 \pm 0.13$ $(-1.34 \pm 0.12)$ for the full (reduced) sample. The values of the spin parameter of the three constrained LGs are $\log _{10}\left(\lambda_{\text {CLUES }}\right)=$ $-2.21,-1.72$, and -1.65 , with the latter value belonging to the sole constrained LG of the reduced sample.

\section{CONCLUSIONS}

We have presented a comparison between the observed kinematics for the M31 in the galactocentric rest frame and the expectations for a large $N$-body cosmological simulation in the $\Lambda \mathrm{CDM}$ cosmology. In the simulation, we select a large sample of pairs that fulfill isolation criteria for the LG. We select a subsample that obeys more stringent observational constraints on the mass and separation of the MW and M31.

While the observations show that M31 moves toward us on a highly eccentric orbit, the simulation shows that the most common configuration at $z=0$ has values $v_{\text {rad, } \Lambda \mathrm{CDM}}=$ $-80 \pm 20 \mathrm{~km} \mathrm{~s}^{-1}$ and $v_{\mathrm{tan}, \Lambda \mathrm{CDM}}=50 \pm 10 \mathrm{~km} \mathrm{~s}^{-1}$, where the error bars are estimated to include the results from the two pair samples.

Using the same absolute values for the uncertainty in the observed velocity components, we find that none of the halos in the reduced sample are compatible with observations. This makes pairs with the preferred $\Lambda \mathrm{CDM}$ values at least 13 times more common than pairs compatible with the observational constraint. Additionally, pairs with a fraction of tangential to radial velocity $f_{\mathrm{t}}<0.32$ (similar to observations) represent $8 \%-12 \%$ of the pairs.

Approximating the LG as two point masses we express the above-mentioned results in terms of the orbital angular momentum $l_{\text {orb }}$ and the mechanical energy $e_{\text {tot }}$ per unit of reduced mass. We find that the uncertainties in the tangential velocity, the square of the norm of the velocity, and the total mass in the LG are less constraining on the number of simulated pairs that are consistent with the observations. Nevertheless, in the case of the reduced sample there is a slight tension between simulation and observation. A reduction by a factor of two in the observational uncertainty on the radial velocity would clarify this issue.

We also use the $\lambda$ spin parameter to gauge the dynamical state of pairs. The values for the orbital angular momentum and energy, merged into the $\lambda$ spin parameter, are in mild disagreement with the observational constraints.

In the three pairs from constrained simulations, we find kinematics dominated by radial velocities. However, their velocity components differ from the observational constraints and their mechanical energy and orbital angular momentum are in broad concordance with observations. There is only one pair that fulfills all the separation, total mass constraints, and matches the most probable value for the dimensionless spin parameter $\lambda$ inferred from observations.
Summarizing, we see a broad agreement in the total angular momentum and energy and a marked difference with the precise balance between today's radial and tangential velocities, i.e., head-on collisions of MW-like halos, as described by $1 \sigma$ uncertainties in observations, are not common in $\Lambda \mathrm{CDM}$.

Under the approximation of conservation of the orbital angular momentum and mechanical energy, this could only be explained if the initial conditions for the formation of the LG are special in comparison to the initial conditions of any other pair of DM halos in the $\Lambda \mathrm{CDM}$ cosmology. Investigating this perspective by a thorough characterization of the small pair sample consistent with observations in terms of halo properties such as concentration, spin, and place in the cosmic web (Forero-Romero et al. 2009; Hoffman et al. 2012) is underway (S. Bustamante et al., in preparation).

The results presented in this Letter open a new window into the question of how unique, if at all, is the LG in a cosmological context. This will continue to be studied within the framework of the CLUES project.

J.E.F.-R. acknowledges financial support from Universidad de los Andes through its Fondo de Apoyo a Profesores Asistentes and the Peter and Patricia Gruber Foundation through its fellowship administered by the IAU. J.E.F.-R. also acknowledges early discussions with Alejandro García that motivated this work. Y.H. has been supported by the ISF (1013/12).

G.Y. acknowledges support from MINECO through research grants AYA 200913875 -C03-02, AYA 2012 31101, and Consolider Syec CSD 2007 0050. Support from Comunidad. De Madrid through ASTROMADRID research grant is also acknowledged.

The data and source code and instructions to replicate the results of this Letter can be found here: https://github.com/forero/LG_Kinematics/. The authors thank the IPython community (Pérez \& Granger 2007). The authors also thank Jessica Kirkpatrick for releasing her Python code to make nice plots of 2D histograms.

The MultiDark Database used in this Letter was constructed as part of the activities of the German Astrophysical Virtual Observatory as result of a collaboration between the LeibnizInstitute for Astrophysics Potsdam (AIP) and the Spanish MultiDark Consolider Project CSD2009-00064. The Bolshoi simulation was run on the NASA's Pleiades supercomputer at the NASA Ames Research Center. The CLUES simulation was run at LRZ Munich.

\section{REFERENCES}

Besla, G., Kallivayalil, N., Hernquist, L., et al. 2007, ApJ, 668, 949

Bett, P., Eke, V., Frenk, C. S., et al. 2007, MNRAS, 376, 215

Busha, M. T., Marshall, P. J., Wechsler, R. H., Klypin, A., \& Primack, J. 2011, ApJ, 743, 40

Forero-Romero, J. E., Hoffman, Y., Gottlöber, S., Klypin, A., \& Yepes, G. 2009, MNRAS, 396, 1815

Forero-Romero, J. E., Hoffman, Y., Yepes, G., et al. 2011, MNRAS, 417, 1434

Fouquet, S., Hammer, F., Yang, Y., Puech, M., \& Flores, H. 2012, MNRAS, 427, 1769

Freeman, K., \& Bland-Hawthorn, J. 2002, ARA\&A, 40, 487

Gottlöber, S., Hoffman, Y., \& Yepes, G. 2010, arXiv:1005.2687

Hoffman, Y., Metuki, O., Yepes, G., et al. 2012, MNRAS, 425, 2049

Jarosik, N., Bennett, C. L., Dunkley, J., et al. 2011, ApJS, 192, 14

Karachentsev, I. D., Karachentseva, V. E., Huchtmeier, W. K., \& Makarov, D. I. 2004, AJ, 127, 2031

Kazantzidis, S., Bullock, J. S., Zentner, A. R., Kravtsov, A. V., \& Moustakas, L. A. 2008, ApJ, 688, 254

Klypin, A., Gottlöber, S., Kravtsov, A. V., \& Khokhlov, A. M. 1999a, ApJ, 516,530 
Klypin, A., Kravtsov, A. V., Valenzuela, O., \& Prada, F. 1999b, ApJ, 522,82

Klypin, A. A., Trujillo-Gomez, S., \& Primack, J. 2011, ApJ, 740, 102

Knebe, A., Libeskind, N. I., Doumler, T., et al. 2011, MNRAS, 417, L56

Liu, L., Gerke, B. F., Wechsler, R. H., Behroozi, P. S., \& Busha, M. T. 2011, ApJ, 733,62

McConnachie, A. W., Irwin, M. J., Ibata, R. A., et al. 2009, Natur, 461, 66

Moore, B., Ghigna, S., Governato, F., et al. 1999, ApJL, 524, L19

Peebles, P. J. E. 1971, A\&A, 11, 377

Peebles, P. J. E., \& Nusser, A. 2010, Natur, 465, 565
Pérez, F., \& Granger, B. E. 2007, CSE, 9, 21

Purcell, C. W., \& Zentner, A. R. 2012, JCAP, 12, 007

Ribas, I., Jordi, C., Vilardell, F., et al. 2005, ApJL, 635, L37

Riebe, K., Partl, A. M., Enke, H., et al. 2011, arXiv:1109.0003

Teyssier, M., Johnston, K. V., \& Kuhlen, M. 2012, MNRAS, 426, 1808

Tikhonov, A. V., \& Klypin, A. 2009, MNRAS, 395, 1915

Tollerud, E. J., Boylan-Kolchin, M., Barton, E. J., Bullock, J. S., \& Trinh, C. Q 2011, ApJ, 738, 102

van der Marel, R. P., Fardal, M., Besla, G., et al. 2012, ApJ, 753, 8

van der Marel, R. P., \& Guhathakurta, P. 2008, ApJ, 678, 187 